\title{
PREVALENCE OF MIGRAINE IN OPHTHALMIC, OPD IN INDIA
}

\author{
Rakesh Sharma ${ }^{1}$, Sumit Sharma ${ }^{2}$, Nidhi Tiwari ${ }^{3}$, Ashish Tripathi ${ }^{4}$
}

${ }^{1}$ Associate Professor, Department of Ophthalmology, MIMS.

${ }^{2}$ Assistant Professor, Department of ENT, MIMS.

${ }^{3}$ Senior Resident, Department of Ophthalmology, MIMS.

4Junior Resident, MIMS.

\section{ABSTRACT}

\section{BACKGROUND}

Headache is essentially a multifactorial disorder and occupies significant disease burden on society. One of the important and frequent causes of such headaches is migraine; if this is untreated the occasional headaches may turn to more frequent and chronic form. In this study, we aimed to investigate the prevalence of migraine headaches in the patients who attended Ophthalmic, Psychiatry, ENT OPD to identify trigger factors and to know its impact on the routine activities and to assess their awareness about the disease in the study population.

\section{MATERIALS AND METHODS}

The target population was the patients in the OPD of the above mentioned departments. All patients who were having headache for more than 6 months were included in the study. Anyone with a history less than 6 months was excluded. International classification of headache disorders (ICHD 2) was utilised to confirm the diagnosis of common migraine (without aura). A questionnaire was prepared for all the patients, which were duly filled by all the patients included in our study which covered demographic profile, character of pain, frequency, duration and intensity of the attacks, accompanying factors, trigger factors, relieving factors and family history of migraine.

\section{RESULTS}

Migraine was seen in $42.4 \%$ of patients of the entire headache sample population. There was a female preponderance of $70 \%$. The frequency of attacks were more than 5 per month in $30 \%$ of patients, while weekly and daily attacks were seen in $25 \%$ and $13 \%$ patients respectively. The remaining $12 \%$ could not report any fixed frequency. Of these, $83 \%$ patients felt that their headaches lasted for more than 4 hours, while for $12 \%$ it lasted for days in continuation. Heaviness of head also persisted for days post an acute attack. Headache did not subside completely even after taking analgesics like diclofenac, aceclofenac and tramadol.

\section{CONCLUSION}

Trigger factors are common in migraine patients. The population under study is subject to various stress factors and recognition of trigger factors will achieve headache remission in a vast majority. Trigger factors in our study group were poor sleep, emotional stress, weather changes and head movements. In fact 75\% patients did feel relieved of their headaches after sleep, though improved. Relieving factors like good sleep, vomiting and food intake help to relieve pain. However, one can prevent it by taking prophylactic medications. There are many medicines available with very good results.

\section{KEYWORDS}

Demographic, Migraine, Prophylactic, Triggers.

HOW TO CITE THIS ARTICLE: Sharma R, Sharma S, Tiwari N, et al. Prevalence of migraine in ophthalmic, OPD in India. J. Evolution Med. Dent. Sci. 2016;5(79):5878-5881, DOI: 10.14260/jemds/2016/1326

\section{BACKGROUND}

Headache is a multi-factorial disorder and has significant disease burden on society; it affects work, social and leisure activities and has a great impact on person's quality of life. Nature of the disease leads to decreased attention and thus decreased productivity at work (Chakravarty A. Chronic daily headache: Clinical profile of Indian patients) One of the important and frequent causes of such headaches is migraine. (Ierusalimschy R, Moreira Filho PF). Precipitating factors of migraine attacks in patients with migraine without aura).

Financial or Other, Competing Interest: None.

Submission 01-09-2016, Peer Review 20-09-2016,

Acceptance 26-09-2016, Published 01-10-2016.

Corresponding Author:

Dr. Rakesh Sharma,

592/115 D, Rajiv Nagar Telibagh,

Lucknow-226025, Uttar Pradesh.

E-mail: rakeshsharma1909@gmail.com

DOI: $10.14260 /$ jemds/2016/1326
If this is untreated, the occasional headaches may turn to more frequent and chronic form.

In this study, we aimed to investigate the prevalence of migraine headaches in the patients who attended our ophthalmic, psychiatry and ENT OPD to identify trigger factors and to know its impact on the routine activities and to assess their awareness about the disease in the study population.

\section{MATERIALS AND METHODS}

The study was conducted in Mayo Institute of Medical Sciences, Barabanki (UP), study conducted between $2^{\text {nd }}$ July 2015 to 30 August 30, 2016. The target population was the patients in the OPD of the above-mentioned departments. International classification of headache disorders (ICHD-2) was utilised to confirm the diagnosis of common migraine without aura being the largest contributor to headaches were only included in our study. Classical migraine (with aura) was not included. An inclusion criterion was followed as 
recommended by international headache society and the classification for migraine without aura, which is as follows:

\section{Diagnostic Criteria for Migraine without Aura}

A. At least five attacks in 1 month and fulfilling criteria B-D

B. Headache attacks lasting 4-72 hours [When untreated in adults]

C. Headache has at least two of the following characteristics

1. Unilateral location.

2. Pulsatile quality.

3. Moderate or severe pain intensity.

4. Aggravation by or causing avoidance of routine physical activity.

D. During the headache, at least one of the following [is present]

- Nausea and/or vomiting.

- Photophobia.

- Phonophobia.

E. Not attributable to another disorder.

A detailed questionnaire was prepared for all the patients, which was filled by all the patients included in our study. The questionnaire covered areas of demographics, character of pain, frequency, duration and intensity of the attacks, accompanying factors, trigger factors, family history of migraine or any other illness, their knowledge about the disease. The questionnaire also included their general lifestyle. Data was tabulated and analysed. Results are expressed in $\mathrm{n}=$ numbers and percentage. The study was approved by the Institutional Ethical Committee.

\section{Exclusion Criteria}

All patients who were having headache for less than 6 months and did not follow the guidelines given under the Diagnostic criteria for migraine without aura were excluded and other diagnosed cases of secondary headaches, i.e. refractive headaches, tension headaches due to sinusitis were excluded.

\section{OBSERVATION AND RESULTS}

A total of 250 patients were included in the study; 211 patients confirmed of having chronic headache and agreed to fill the questionnaire; 39 were excluded after evaluating their questionnaire. They had reported vague episode of headache lasting for less than 4 hours, which did not confirm to ICHD-2 criteria (headache attacks lasting 4-72 hours without medication. Of the 211 patients, 106 (50.2\%) fulfilled the criteria for migraine according to ICHD-2 criteria.[1] The rest $(49.8 \%)$ had other forms of headaches, which do not fall under the criteria for common migraine.

Migraine was seen in $42.4 \%$ of patients of the entire headache sample population (106 cases). There was a female preponderance of $70 \%$. Weekly and daily attacks were seen in $25 \%$ and $13 \%$ patients, respectively. The remaining $12 \%$ could not report any fixed frequency. Of these $83 \%$ patients felt that their headaches lasted for hours, while for $17 \%$ it lasted for days in continuation. Heaviness of head also persisted for days post an acute attack. Headache did not subside completely even after taking analgesics.

$51 \%$ of patients felt that their headache had increased in intensity and frequency since the beginning; $45 \%$ of migraine headaches were more than 4 per week. However, only $18 \%$ patients went to emergency services because of the severe headache. Photophobia, phonophobia, nausea, irritability, anxiety and vomiting were the most common accompanying symptoms. Vertigo along with headache was seen in 35\% of patients. Such cases were referred to ENT Department to evaluate for vestibular migraine. The other accompanying factors are problem in sleep initiation, drooping of eyelids, diarrhea, feverishness, drowsiness, speech disturbance, swelling of ankles, tearing of eyes, aura, visual disturbances, transient loss of vision, periodic headache, vertigo, etc. Trigger factors could be identified in all patients; $81 \%$ patients had more than one identifiable trigger ${ }^{[1,2]}$ factors, prominent were stress, poor sleep, environmental changes, head movements and mental stress, fasting, menstrual phase, emotional stress. Other less common identified factors were ocular strain, fasting, exertion, perfumes and journey.

Patients experienced relief from migraine after sleep (75\%), vomiting (16\%), tying head with cloth (5\%) and with food intake (4\%).[3,4] Responses about lifestyle revealed that one-fourth of the patients slept for less than 6 hours. This may be why females are more affected. Level of physical activity assessment showed that only $11 \%$ of patients did daily exercise, $51 \%$ did mild-to-moderate form of exercise infrequently; $38 \%$ did not have any form of exercise. The most common exercise was walking; $50 \%$ percent of patients frequently missed meals; $50 \%$ of patients used analgesics whenever required. None of them had any prophylactic medicines. Family history of migraine was seen in 30\%, specially the females, ${ }^{[5,2]}$ while a large proportion of patients (49\%) did not feel the need for any consultation. Thirty percent of patients attributed their headache to stress, while only a small proportion (10\%) attributed it to migraine. Headache aggravated by routine activities was seen in $81 \%$ of patients, $23 \%$ had decreased capability; $18 \%$ of patients had to miss family, social and leisure activities.

\section{Common Symptoms and Association}

Headache is a common complaint across all age groups and recurrent headaches causes a considerable burden on the individual as well as the society. Headache affects social life, $40 \%$ of the patients complaining of headache in our study group conformed to the diagnosis of migraine elsewhere. Migraine was more than twice as frequent in females being $70 \%$ as compared with $30 \%$ of male patients as reported in earlier studies.

The most characteristic headache was pulsatile or the throbbing headache, hemi-headache involving temple region (ICHD-2), which was seen in $85 \%$ of patients. The impact of migraine on a person can be assessed by the frequency of pain, severity and the accompanying factors. However, only onefourth of them approached health services for treatment.

In addition to pain, associated symptoms were common. Our study showed photophobia, phonophobia, nausea and vomiting as the most common accompanying symptoms reported previously (ICHD-2). Other reported symptoms were dizziness, allodynia and neck stiffness. These accompanying symptoms increase the disability of the patient and are occasionally more troublesome for the patient. Indeed, if accompanying symptoms are more prominent than headache, known as migraine variant, the diagnosis can be difficult delaying the treatment. Recent study found that $63 \%$ of individuals with migraine and tension-type headache had muscular pain around the neck and shoulder region. Neck pain was found to be a commoner symptom than the habitual 
nausea, and perhaps awareness of this symptom will definitely increase the diagnostic accuracy.

One-third of the patients also had vertigo with migraine, raising the possibility of vestibular migraine. However, we could not be definite whether it was a comorbid phenomenon, given the fact that both symptoms are very common in the general population. Vestibular migraine is the second common cause of vertigo and a common cause of spontaneous vertigo. The presence of vertigo is a diagnostic challenge, as occasionally vestibular migraine can present with vertigo and other features without headache. Allodynia (perception of non-painful stimuli as painful) was reported by $23 \%$ of patients. We considered only the acute allodynia and not the interictal form. Patients reported scalp tenderness while combing hair and facial tenderness during migraine attacks. The presence of allodynia has been associated with more frequent, severe and disabling headaches. Presence of allodynia is a clinical correlate to central sensitisation. Hence, the recognition of allodynia by the patient and the treating physician is crucial, as these patients respond to Triptans before or soon after the onset of allodynia.

$10 \%$ percent of the patients had history of motion sickness in the past. There is evidence of motion sickness being a comorbid phenomenon in migraine. Whether individuals with motion sickness have heightened susceptibility to develop migraine later in life is to be answered.

\section{Triggering Factors}

\begin{tabular}{|c|c|c|}
\hline $\begin{array}{c}\text { Emotional Liability and Stress } \\
\text { (Physical and Mental) }\end{array}$ & 86 & $81 \%$ \\
\hline Sleep disturbance & 81 & $76 \%$ \\
\hline Head movements & 73 & $68 \%$ \\
\hline Environmental factors & 67 & $63 \%$ \\
\hline Fasting & 67 & $63 \%$ \\
\hline Hair wash & 59 & $55 \%$ \\
\hline Menstrual phase & 58 & $54 \%$ \\
\hline Ocular strain & 32 & $30 \%$ \\
\hline Journey & 32 & $30 \%$ \\
\hline Perfumes & 22 & $20 \%$ \\
\hline Specific food & 20 & $18 \%$ \\
\hline Beverages & 17 & $15 \%$ \\
\hline Table 1: Num (n) Percent & \\
\hline
\end{tabular}

Trigger factors in decreasing order of frequency.

\section{DISCUSSION}

Trigger factors are common in migraine patients (Yadav RK, A study of triggers of migraine in India). The population under study is subject to various stress factors and recognition of trigger factors will achieve headache remission in a vast majority. (Ierusalimschy $\mathrm{R}$ et al, Precipitating factors of migraine attacks in patients with migraine without aura). Trigger factors in our study group were poor sleep, emotional stress, weather changes and head movements. In fact, $75 \%$ patients did feel relieved of their headaches after sleep. Previous studies have reported sleep disturbance and emotional stress as triggers for migraine.

A few other studies (Kimoto K, Aiba S, Takashima R, Suzuki $\mathrm{K}$, Takekawa $\mathrm{H}$, Watanabe $\mathrm{Y}$, et al. Influence of barometric pressure in patients with migraine headache) have shown weather and barometric change as trigger factors. Head and neck movement could be seen as cervical triggers, ${ }^{[6]}$ which increase the peripheral sensitisation from the cervical afferents. Active trigger points in the head and neck areas can be involved in the initiation and propagation of migraine. Apart from being trigger factors, head and neck movements have also been shown to aggravate pain.[7] Odors as a trigger factor were seen in $20 \%$ of patients, which has been described in the past. Olfactory hallucination or osmophobia can occur as a symptom complex in migraine. Our study also documented a previously reported head bath as a trigger factor in the Indian population in $60 \%$ of our patients. Bath-Related Headache (BRH) is a rare primary headache syndrome. Hair wash or head bath has been found as an unusual trigger for migraine, specially in females, specially during the winter season. (Ravishankar K. Hair-wash headache - an unusual trigger for migraine in Indian patients). However, the pathophysiology behind the trigger has not been identified.

Trigger factors vary with type of population under study and the geographical area (Yadav RK et al. A study of triggers of migraine in India), and one needs to be aware of them for effective counseling for migraine patients. The modifiable trigger factors in the study group were sleep pattern, emotional stress, head movements and resorting to timely food intake; non-modifiable triggers were weather changes, menstrual cycles, hair wash and journey. The same may be with the custom of hair wash in India (Ravishankar K. Hairwash headache - an unusual trigger for migraine in Indian patients), which probably may be difficult to modify.

\section{Relieving Factors}

\begin{tabular}{|c|c|}
\hline Sleep & $75 \%$ \\
\hline Vomiting & $16 \%$ \\
\hline Tying head & $5 \%$ \\
\hline Food intake & $4 \%$ \\
\hline
\end{tabular}

However, one can prevent it by taking prophylactic medications. There are many medicines available in the market with very good success rates.

- Flunarizine.

- Nortriptyline.

- Amitriptyline.

- Topiramate.

- Propranolol.

- Atenolol.

- Metoprolol.

The goals of preventive therapy are to reduce the frequency, pain and duration of migraines, and to increase quality of life.

Moreover our study showed that there was clustering of trigger factors; addressing each trigger could considerably reduce the frequency of attacks.

Family history of headache was seen in 30\% patients, previous studies reporting between $22.0 \%$ and $80 \%$. A section of the questionnaire was regarding the lifestyle of the patients. We found an alarmingly low $11 \%$ patients only did exercise on a daily basis. Patients with low physical activity are at risk of recurrent headaches. In fact, a recent study also concluded exercise as an alternative to prophylactic treatment of migraine. So to have the educational curriculum include physical exercise or games, which will help prevent headaches and also other fatal diseases. 
Migraine-related disability had a few limitations. We did not address the aura component and did not sub-classify the migraine forms. However, these shortcomings do not affect the main purpose of the study.

The awareness of the disease was very low. There were identifiable triggers in the population, which can be tailored for the treatment. This study will further help primary care providers to address patient education in this population as well as to assist patients in properly managing their headaches. Further larger studies should be planned to have more in-depth knowledge of migraine in the various population.

\section{CONCLUSION}

Headache is a frequent complaint in the patients coming to ophthalmic OPD, a significant majority of patients have migrainous headache (Ierusalimschy R, Moreira Filho PF. Precipitating factors of migraine attacks in patients with migraine without aura). In our study population, there were presence of trigger factors and relieving factors of migraine. If the patient knows about these factors which in majority of the patients are modifiable, the frequency and severity of headache episodes may be prevented. The modifiable trigger factors in the study group were sleep pattern, emotional stress, head movements and resorting to timely food intake; non-modifiable triggers were weather changes, menstrual cycles, hair wash and journey. Relieving factors are important as they decrease the severity of pain. Migraine-related disability had a few limitations. We did not address the aura component and did not sub-classify the migraine forms. However, these shortcomings do not affect the main purpose of the study.

At last the prophylactic medicines have significantly decreased the frequency and the severity of the headaches.

\section{REFERENCES}

1. Headache classification subcommittee of international headache society. International classification of headache disorders $2^{\text {nd }}$ edition. Cephalalgia 2004;24(Suppl 1):9-160.

2. Blaschek A, Milde-Busch A, Straube A, et al. Self-reported muscle pain in adolescents with migraine and tension-type headache. Cephalalgia 2012;32(3):241-9.

3. Yadav RK, Kalita J, Misra UK. A study of triggers of migraine in India. Pain Med 2010;11(1):44-7.

4. Ravishankar K. Hair-wash headache-an unusual trigger for migraine in Indian patients. Cephalalgia 2005; 25(12): 1184-5.

5. Bokhari FA, Sami W, Shakoori TA, et al. Clinical characteristics of 226 college-going female migraineurs in Lahore, Pakistan - putting ICHD-2 to the road test. Neuro Endocrinol Lett 2008;29(6):965-70.

6. Fernández-de-Las-Peñas C, Cuadrado ML, Pareja JA. Myofascialtrigger points, neck mobility and forward head posture in unilateral migraine. Cephalalgia 2006;26(9): 1061-70.

7. Ierusalimschy R, Moreira Filho PF. Precipitating factors of migraine attacks in patients with migraine without aura. Arq Neuropsiquiatr 2002;60(3-A):609-13. 